QCD Evolution Workshop 2013

International Journal of Modern Physics: Conference Series

Vol. 25 (2014) 1460024 (11 pages)

(C) The Author

DOI: $10.1142 / \mathrm{S} 2010194514600246$

\title{
NLO BFKL AND ANOMALOUS DIMENSIONS OF LIGHT-RAY OPERATORS*
}

\author{
IAN BALITSKY \\ Physics Department, Old Dominion University, Norfolk VA 23529 \\ and \\ Theory Center, Jefferson Lab, Newport News, VA 23606,USA \\ balitsky@jlab.org
}

Published 2 January 2014

\begin{abstract}
The anomalous dimensions of light-ray operators of twist two are obtained by analytical continuation of the anomalous dimensions of corresponding local operators. I demonstrate that the asymptotics of these anomalous dimensions at the "BFKL point" $j \rightarrow 1$ can be obtained by comparing the light-cone operator expansion with the high-energy expansion in Wilson lines.
\end{abstract}

Keywords: High energy; Anomalous dimensions; Wilson lines.

PACS numbers: 12.38.Bx, 12.38.Cy

\section{Introduction}

It is well known that the BFKL pomeron ${ }^{1}$ gives the anomalous dimensions of leading-twist gluon operators at all orders near the unphysical point with number of covariant derivatives equal to minus one. The exact statement is that the analytical continuation of anomalous dimension of twist-two gluon operator

$$
\mathcal{O}_{F}^{n} \equiv F_{\mu-}^{a} \nabla_{-}^{j-2} F_{-}^{\mu a}
$$

to the point $j=1$ is determined by the BFKL equation. The anomalous dimensions are singular at that point and there is a new hierarchy of perturbation theory $\gamma\left(\alpha_{s}, n\right) \sim \sum \alpha_{s}^{m}\left(\frac{\alpha_{s}}{j-1}\right)^{n-m}$. In the leading order $\sim\left(\frac{\alpha_{s}}{j-1}\right)^{n}$ the relation to the BFKL pomeron was established long ago $^{2}$ and for the next-to-leading order BFKL it was done in papers [3]. This constitutes a powerful check for explicit calculations of higher-order anomalous dimensions both in QCD [4] and $\mathcal{N}=4$ SYM [5]. However, the explicit meaning of these "local operators at the unphysical point"

*This is an Open Access article published by World Scientific Publishing Company. It is distributed under the terms of the Creative Commons Attribution 3.0 (CC-BY) License. Further distribution of this work is permitted, provided the original work is properly cited. 


\section{Balitsky}

was somewhat obscure. I argue that these operators should be understood as gluon light-ray operators and BFKL equation gives the anomalous dimensions of these light-ray operators at some specific point.

First, let me remind the standard argument about analytical continuation. It is well known that the moments of structure functions are proportional to matrix elements of twist- 2 operators. The $Q^{2}$ behavior of structure functions are governed by anomalous dimensions of these operators

$$
\mu \frac{d}{d \mu} F_{\mu-}^{a} \nabla_{-}^{n-2} F_{-}^{\mu a}=\gamma\left(\alpha_{s}, n\right) F_{\mu-}^{a} \nabla_{-}^{n-2} F_{-}^{\mu a}
$$

As I mentioned, the BFKL pomeron determines the asymptotics of these anomalous dimension at the "non-physical" point $n=1$. The standard argument goes like that: in the framework of the BFKL approach the amplitude of, say, virtual $\gamma^{*} \gamma^{*}$ scattering has the form

$$
A(s)=\int d \nu F(\nu)\left(\frac{s}{Q P}\right)^{\aleph(\nu)}\left(\frac{Q^{2}}{P^{2}}\right)^{i \nu}
$$

where $\aleph(\nu)$ is the pomeron intercept (the explicit form is given by Eq. (41) below) where $Q^{2}=-q^{2}$ and $p^{2}=-P^{2}$ are virtualities of the two photons and $s=(p+q)^{2}$. The case of DIS corresponds to $Q^{2} \gg P^{2}$ and the Regge limit of small-x DIS to $s \gg Q^{2}$

Rewriting this in terms of Bjorken $x_{B}=Q^{2} / s$ one gets

$$
A(s)=\int d \nu F(\nu) x_{B}^{-\aleph(\nu)}\left(\frac{Q^{2}}{P^{2}}\right)^{i \nu+\frac{1}{2} \aleph(\nu)}
$$

which turns to

$$
A\left(x_{B}, Q^{2}\right)=\frac{s}{Q P} \int d \nu F(\nu) x_{B}^{-\tilde{\aleph}(\nu)}\left(\frac{Q^{2}}{P^{2}}\right)^{i \nu}
$$

after the shift

$$
\nu-\frac{i}{2} \aleph(\nu) \rightarrow \nu
$$

The $n$-th moment of the structure function is given by

$$
M_{n}=\int_{0}^{1} d x_{B} x_{B}^{n} A\left(x_{B}, Q^{2}\right)=\int_{\frac{1}{2}-i \infty}^{\frac{1}{2}+i \infty} d \xi \frac{F(\xi)}{n-\tilde{\aleph}(\xi)}\left(\frac{Q^{2}}{P^{2}}\right)^{\xi}
$$

where $\xi \equiv \frac{1}{2}+i \nu$. Now let us consider the integral (6) at $n=\omega \rightarrow 0$. The contour goes parallel to imaginary axis but we can close it on the poles of the function $\frac{1}{\omega-\tilde{\aleph}(\xi)}$. The expansion of $\tilde{\aleph}(\xi)$ at small $\xi$ has the form

$$
\tilde{\aleph}(\xi)=\frac{\alpha_{s} N_{c}}{2 \pi \xi}+\frac{\alpha_{s}^{2} N_{c}^{2}}{8 \pi^{2} \xi} \zeta(3)+O\left(\alpha_{s}^{3}\right)
$$


so we get

$$
M_{\omega}=\int_{0}^{1} d x_{B} x_{B}^{\omega} A\left(x_{B}, Q^{2}\right)=\left(\frac{Q^{2}}{P^{2}}\right)^{\gamma(\omega)}
$$

where

$$
\left.\gamma\left(\alpha_{s}, \omega\right)=-2 \frac{\alpha_{s} N_{c}}{\pi \omega}+[0+\zeta(3) \omega]\left(\frac{\alpha_{s} N_{c}}{\pi \omega}\right)\right)^{3}+\ldots
$$

Since

$$
M_{n}=\int_{0}^{1} d x_{B} x_{B}^{n} A\left(x_{B}, Q^{2}\right)=\left(\frac{Q^{2}}{P^{2}}\right)^{\gamma_{n}}
$$

we see that the series (8) giver the analytical continuation of the anomalous dimensions of twist-2 operators (1) at the point $n \rightarrow 1$ which can be formally denoted as $F_{-i} \nabla^{-1} F_{-}^{i}$.

This method, however, does not tell us the explicit form of this operator and in this paper I will demonstrate that $F_{-i} \nabla^{\omega-1} F_{-}{ }^{i}$ is actually a light-ray operator $(j \equiv \omega+1)$

$$
\mathcal{F}_{j}\left(x_{\perp}\right)=\int_{0}^{\infty} d L_{+} L_{+}^{1-j} \int d x_{+} F_{-i}^{a}\left(L_{+}+x_{+}+x_{\perp}\right)\left[L_{+}+x_{+}, x_{+}\right]^{a b} F_{-}^{b i}\left(x_{+}+x_{\perp}\right)
$$

and the anomalous dimension of this operator $\gamma\left(j ; \alpha_{s}\right)$ is an analytic continuation of the anomalous dimension (2) of local operators.

For simplicity this will be done in $\mathcal{N}=4$ SYM where the conformal invariance simplifies many formulas for the correlation functions. The method to get the anomalous dimensions of the light-ray operator (10) near $j=1$ will be to calculate a 4-point correlation function of certain operators in the double "Regge+DIS" limit $s \gg Q^{2} \gg P^{2}$. We will compare two calculations of this 4-point CF: with Regge limit first and DGLAP limit second, or vice versa, and demonstrate that the intercept of the BFKL pomeron $\aleph(\nu)$ determines the anomalous dimensions in this region. However, first we need to discuss some properties of light-ray operators.

\section{Light-Ray Operators}

\subsection{Light-ray operators in gluodynamics}

For simplicity, let us at first look at gluodynamics. For our purposes it is sufficient to consider "forward" light-ray operator

$$
F\left(L_{+}, x_{\perp}\right)=\int d x_{+} F_{-i}^{a}\left(L_{+}+x_{+}+x_{\perp}\right)\left[L_{+}+x_{+}, x_{+}\right]^{a b} F_{-}^{b i}\left(x_{+}+x_{\perp}\right)
$$

Evolution equation for "forward" operators has the form (see e.g. Ref. [6])

$$
\mu \frac{d}{d \mu} F\left(L_{+}, x_{\perp}\right)=\int_{0}^{1} d u K_{g g}\left(u, \alpha_{s}\right) F\left(u L_{+}, x_{\perp}\right)
$$




\section{Balitsky}

Note that since the matrix element of the light-ray operator (11) defines gluon parton density

$$
z^{\mu} z^{\nu}\left\langle p\left|F_{\mu \xi}^{a}(z)[z, 0]^{a b} F_{\nu}^{b \xi}(0)\right| p\right\rangle^{\mu} \stackrel{z^{2}=0}{=} 2(p z)^{2} \int_{0}^{1} d x_{B} x_{B} D_{g}\left(x_{B}, \mu\right) \cos (p z) x_{B}
$$

the kernel

$$
u^{-1} K_{g g}(u)=\frac{2 \alpha_{s} N_{c}}{\pi}\left(\bar{u} u+\left[\frac{1}{\bar{u} u}\right]_{+}-2+\frac{11}{12} \delta(\bar{u})\right)+\text { higher orders in } \alpha_{s}
$$

is actually a DGLAP kernel (in gluodynamics).

The anomalous dimensions of local twist-2 operators (1) is determined by the kernel (13)

$$
\gamma_{n}\left(\alpha_{s}\right)=-\int_{0}^{1} d u u^{n-2} K_{g g}\left(u, \alpha_{s}\right) \quad \mu \frac{d}{d \mu} \mathcal{O}_{n}^{g}=-\gamma_{n}\left(\alpha_{s}\right) \mathcal{O}_{n}^{g}
$$

Now consider the anomalous dimensions of LR operator (10). Cobining Eqs. (10) and (12) one obtains

$$
\mu \frac{d}{d \mu} \mathcal{F}_{j}\left(z_{\perp}\right)=\int_{0}^{1} d u K_{g g}\left(u, \alpha_{s}\right) u^{j-2} \mathcal{F}_{j}\left(z_{\perp}\right)
$$

Thus, we see that the anomalous dimension of light-ray operators (11) is an analytical continuation of anomalous dimensions of local operators to non-integer $j$.

\subsection{Singlet light-ray operators in $\mathcal{N}=4 S Y M$}

In $\mathcal{N}=4 \mathrm{SYM}$, in addition to the gluon operators (1) there are twist-2 local gluino and scalar operators (as usual, we consider only "forward" highest weight local operators). Similarly to Eq. (11) we can define gluino and scalar light-ray operators (of even parity)

$$
\begin{aligned}
\Phi\left(x_{+}, x_{\perp}\right)= & \int d x_{+}^{\prime} \phi_{I}^{a}\left(x_{+}+x_{+}^{\prime}+x_{\perp}\right)\left[x_{+}, x_{+}^{\prime}\right]^{a b} \nabla_{-}^{2} \phi_{I}^{b}\left(x_{+}^{\prime}+x_{\perp}\right) \\
\Lambda\left(x_{+}, x_{\perp}\right)= & \frac{i}{2} \int d x_{+}^{\prime}\left[\bar{\lambda}_{A}^{a}\left(x_{+}+x_{+}^{\prime}+x_{\perp}\right)\left[x_{+}+x_{+}^{\prime}, x_{+}^{\prime}\right]^{a b} \sigma_{-} \nabla_{-} \lambda_{A}^{b}\left(x_{+}^{\prime}+x_{\perp}\right)\right. \\
& \left.-\bar{\lambda}_{A}^{a}\left(x_{+}^{\prime}+x_{\perp}\right)\left[x_{+}+x_{+}^{\prime}, x_{+}\right]^{a b} \sigma_{-} \nabla_{-} \lambda_{A}^{b}\left(x_{+}+x_{+}^{\prime}+x_{\perp}\right)\right] \\
G\left(x_{+}, x_{\perp}\right)= & \int d x_{+}^{\prime} F_{-i}^{a}\left(x_{+}+x_{+}^{\prime}+x_{\perp}\right)\left[x_{+}+x_{+}^{\prime}, x_{+}^{\prime}\right]^{a b} F_{-}^{b i}\left(x_{+}^{\prime}+x_{\perp}\right)
\end{aligned}
$$

The evolution equations have the form similar to (12)

$$
\begin{aligned}
\mu \frac{d}{d \mu} \Phi\left(x_{+}\right) & =\int_{0}^{1} d u\left[K_{\phi \phi} \Phi\left(u x_{+}\right)+K_{\phi \lambda} \Lambda\left(u x_{+}\right)+K_{\phi g} G\left(u x_{+}\right)\right] \\
\mu \frac{d}{d \mu} \Lambda\left(x_{+}\right) & =\int_{0}^{1} d u\left[K_{\lambda \phi} \Phi\left(u x_{+}\right)+K_{\lambda \lambda} \Lambda\left(u x_{+}\right)+K_{\lambda g} G\left(u x_{+}\right)\right] \\
\mu \frac{d}{d \mu} G\left(x_{+}\right) & =\int_{0}^{1} d u\left[K_{g \phi} \Phi\left(u x_{+}\right)+K_{g \lambda} \Lambda\left(u x_{+}\right)+K_{g g} G\left(u x_{+}\right)\right]
\end{aligned}
$$


where $K_{i j}=K_{i j}\left(u, \alpha_{s}\right)$ is a function of $u$ and $\alpha_{s}$. The expansion in powers of $x_{+}$ gives anomalous dimensions of local operators

$$
\begin{aligned}
\Phi\left(x_{+}, x_{\perp}\right) & =\sum \frac{x_{+}^{n-2}}{(n-2) !} \mathcal{O}_{\phi}^{n}\left(x_{\perp}\right), \quad \Lambda\left(x_{+}, x_{\perp}\right)=\sum \frac{x_{+}^{n-2}\left(x_{\perp}\right)}{(n-2) !} \mathcal{O}_{\lambda}^{n}, \\
G\left(x_{+}, x_{\perp}\right) & =\sum \frac{x_{+}^{n-2}}{(n-2) !} \mathcal{O}_{g}^{n}\left(x_{\perp}\right)
\end{aligned}
$$

where

$$
\begin{aligned}
\mathcal{O}_{\phi}^{n}\left(x_{\perp}\right) & =\int d x_{+}^{\prime} \bar{\phi}_{A B}^{a} \nabla_{-}^{n} \phi^{A B a}\left(x_{+}^{\prime}+x_{\perp}\right), \\
\mathcal{O}_{\lambda}^{n}\left(x_{\perp}\right) & =\int d x_{+}^{\prime} i \bar{\lambda}_{A}^{a} \nabla_{-}^{n-1} \sigma_{-} \lambda_{A}^{a}\left(x_{+}^{\prime}+x_{\perp}\right) \\
\mathcal{O}_{g}^{n}\left(x_{\perp}\right) & =\int d x_{+}^{\prime} F_{-i}^{a} \nabla_{-}^{n-2} F_{-}^{a i}\left(x_{+}^{\prime}+x_{\perp}\right),
\end{aligned}
$$

Matrix of anomalous dimensions for these "forward" local operators has the form

$$
\begin{aligned}
\mu \frac{d}{d \mu} \mathcal{O}_{\phi}^{n} & =\int_{0}^{1} d u u^{n-2}\left[K_{\phi \phi} \mathcal{O}_{\phi}^{n}+K_{\phi \lambda} \mathcal{O}_{\lambda}^{n}+K_{\phi g} \mathcal{O}_{g}^{n}\right] \\
\mu \frac{d}{d \mu} \mathcal{O}_{\lambda}^{n} & =\int_{0}^{1} d u u^{n-2}\left[K_{\lambda \phi} \mathcal{O}_{\phi}^{n}+K_{\lambda \lambda} \mathcal{O}_{\lambda}^{n}+K_{\lambda g} \mathcal{O}_{g}^{n}\right] \\
\mu \frac{d}{d \mu} \mathcal{O}_{g}^{n} & =\int_{0}^{1} d u u^{n-2}\left[K_{g \phi} \mathcal{O}_{\phi}^{n}+K_{g \lambda} \mathcal{O}_{\lambda}^{n}+K_{g g} \mathcal{O}_{g}^{n}\right]
\end{aligned}
$$

The renorm-invariant combinations were found in Ref. [7]:

$$
\begin{aligned}
S_{1}^{n} & =\mathcal{O}_{g}^{n}+\frac{1}{4} \mathcal{O}_{\lambda}^{n}-\frac{1}{2} \mathcal{O}_{\phi}^{n}, \quad S_{2}^{n}=\mathcal{O}_{g}^{n}-\frac{1}{4(n-1)} \mathcal{O}_{\lambda}^{n}+\frac{(n+1)}{6(n-1)} \mathcal{O}_{\phi}^{n} \\
S_{3}^{n} & =\mathcal{O}_{g}^{n}-\frac{n+2}{2(n-1)} \mathcal{O}_{\lambda}^{n}-\frac{(n+1)(n+2)}{2 n(n-1)}
\end{aligned}
$$

The corresponding anomalous dimensions read

$$
\gamma_{n}^{S_{1}}\left(\alpha_{s}\right)=4\left[\psi(n-1)+\gamma_{E}\right]+O\left(\alpha_{s}^{2}\right), \quad \gamma_{n}^{S_{2}}=\gamma_{n+1}^{S_{1}}, \quad \gamma_{n}^{S_{3}}=\gamma_{n+2}^{S_{1}}
$$

Now we define

$$
\begin{aligned}
\Phi_{j}\left(x_{\perp}\right) & =\int_{0}^{\infty} d x_{+} x_{+}^{-j+1} \Phi\left(x_{+}, x_{\perp}\right), \quad \Lambda_{j}\left(x_{\perp}\right)=\int_{0}^{\infty} d x_{+} x_{+}^{-j+1} \Lambda\left(x_{+}, x_{\perp}\right) \\
G_{j}\left(x_{\perp}\right) & =\int_{0}^{\infty} d x_{+} x_{+}^{-j+1} G\left(x_{+}, x_{\perp}\right)
\end{aligned}
$$

From Eq. (16) we get the martix of anomalous dimensions for these LR opertaors

$$
\begin{aligned}
\mu \frac{d}{d \mu} \Phi_{j} & =\int_{0}^{1} d u u^{j-2}\left[K_{\phi \phi}\left(u, \alpha_{s}\right) \Phi_{j}+K_{\phi \lambda}\left(u, \alpha_{s}\right) \Lambda_{j}+K_{\phi g}\left(u, \alpha_{s}\right) G_{j}\right] \\
\mu \frac{d}{d \mu} \Lambda_{j} & =\int_{0}^{1} d u u^{j-2}\left[K_{\lambda \phi}\left(u, \alpha_{s}\right) \Phi_{j}+K_{\lambda \lambda}\left(u, \alpha_{s}\right) \Lambda_{j}+K_{\lambda g}\left(u, \alpha_{s}\right) G_{j}\right] \\
\mu \frac{d}{d \mu} G_{j} & =\int_{0}^{1} d u u^{j-2}\left[K_{g \phi}\left(u, \alpha_{s}\right) \Phi_{j}+K_{g \lambda}\left(u, \alpha_{s}\right) \Lambda_{j}+K_{g g}\left(u, \alpha_{s}\right) G_{j}\right]
\end{aligned}
$$




\section{Balitsky}

Since the matrix (23) is an analytical continuation of the matrix (16) so are the eigenvectors and eigenfunctions and therefore

$$
\begin{aligned}
& S_{1}^{j}=G_{j}+\frac{1}{4} \Lambda_{j}-\frac{1}{2} \Phi_{j}, \quad S_{2}^{j}=G_{j}-\frac{1}{4(j-1)} \Lambda_{j}+\frac{j+1}{6(j-1)} \Phi_{j} \\
& S_{3}^{j}=G_{j}-\frac{j+2}{2(j-1)} \Lambda_{j}-\frac{(j+1)(j+2)}{2 j(j-1)} \Phi_{j}
\end{aligned}
$$

are the multiplicatively renormalized operators with anomalous dimensions

$$
\gamma_{j}^{S_{1}}\left(\alpha_{s}\right)=4\left[\psi(j-1)+\gamma_{E}\right]+O\left(\alpha_{s}^{2}\right), \quad \gamma_{j}^{S_{2}}=\gamma_{j+1}^{S_{1}}, \quad \gamma_{j}^{S_{3}}=\gamma_{j+2}^{S_{1}}
$$

Note that at high energy $\left(\equiv j \rightarrow 1\right.$ ) only the contribution of $S_{1}$ survives because at small $\omega=j-1$ the anomalous dimension of $S_{1}$ is negative while those of $S_{2}$ and $S_{3}$ are non-negative and hence we omit contributions of $S_{2}$ and $S_{3}$ operators in what follows.

For future use we need also the operators with the light rays going along the $x_{-}$ direction defined as

$$
\begin{aligned}
\tilde{\Phi}_{j}\left(x_{\perp}\right) & =\int_{0}^{\infty} d x_{-} x_{-}^{-j-1} \Phi\left(x_{-}, x_{\perp}\right) \\
& =\int d x_{-} d x_{-}^{\prime} \theta\left(x_{-}\right) x_{-}^{-j-1} \bar{\phi}_{A B}^{a}\left(x_{-}+x_{-}^{\prime}+x_{\perp}\right)\left[x_{-}, x_{-}^{\prime}\right]^{a b} \phi^{A B b}\left(x_{-}^{\prime}+x_{\perp}\right)
\end{aligned}
$$

and similarly for $\tilde{\Lambda}_{j}, \tilde{G}_{j}$ and $\tilde{S}_{j}$ operators.

\section{DGLAP Representation of 4-Point Correlation Function}

To get the anomalous dimensions of light-ray operators (24) near $j=1$ we consider the correlation function of four Konishi operators in the double BFKL + DGLAP limit. Define

$$
A\left(x_{1}, x_{2}, x_{3}, x_{4}\right)=\mu^{-4}\left(\mu^{4} x_{12}^{2} x_{34}^{2}\right)^{2+\gamma_{k}}\left\langle\mathcal{O}\left(x_{1}\right) \mathcal{O}\left(x_{2}\right) \mathcal{O}\left(x_{3}\right) \mathcal{O}\left(x_{4}\right)\right\rangle
$$

where $\mathcal{O}=\phi_{I}^{a} \phi_{I}^{a}$ is the Konishi operator $\left(\gamma_{K}\right.$ - anomalous dimension). The Regge limit is

$$
x_{1+} \rightarrow \rho x_{1+}, x_{2+} \rightarrow \rho x_{2+}, \quad x_{3-} \rightarrow \rho^{\prime} x_{3-}, x_{4-} \rightarrow \rho^{\prime} x_{4-} \quad \text { with } \quad \rho \rho^{\prime} \rightarrow \infty
$$

and the DGLAP limit corresponds to $x_{12}^{2} \rightarrow 0$. It is convenient to consider "forward" correlation function

$$
\begin{aligned}
& A\left(L_{+}, L_{-} ; x_{1_{\perp}}, x_{2_{\perp}}, x_{3_{\perp}}, x_{4_{\perp}}\right)=\mu^{-4}\left(\mu^{4} x_{12}^{2} x_{34}^{2}\right)^{2+\gamma_{k}} \\
& \quad \times \int d x_{2_{+}} d x_{3_{-}}\left\langle\mathcal{O}\left(L_{+}+x_{2_{+}}, x_{1_{\perp}}\right) \mathcal{O}\left(x_{2_{+}}, x_{2_{\perp}}\right) \mathcal{O}\left(L_{-}+x_{4_{-}}, x_{3_{\perp}}\right) \mathcal{O}\left(x_{4_{-}}, x_{4_{\perp}}\right)\right\rangle
\end{aligned}
$$

in the double limit: Regge $\left(L_{+} L_{-} \rightarrow \infty\right)$ plus DGLAP $\left(x_{12}^{2} \rightarrow 0\right)$.

First we look at the CF (29) in the DGLAP limit. In this limit we need to expand of the product two Konishi operators near the light cone with the leading term being twist-two light-ray operators (24). To get the coefficient functions in 
this expansion it is convenient to discuss first the three-point correlation function of a LR operator with two local operators. The correlation function of three local operators is fixed by conformal invariance. In our case it yields

$$
\frac{\left\langle S_{1 n}\left(z_{1}\right) \mathcal{O}\left(z_{2}\right) \mathcal{O}\left(z_{3}\right)\right\rangle}{\left(\mu^{2} z_{23}^{2}\right)^{\gamma_{K}}}=\frac{c_{n}\left[1+(-1)^{n}\right]}{z_{12}^{2} z_{13}^{2} z_{23}^{2}}\left(\frac{z_{12_{-}}}{z_{12}^{2}}-\frac{z_{13_{-}}}{z_{13}^{2}}\right)^{n}\left(\frac{\mu^{-2} z_{23}^{2}}{z_{12}^{2} z_{13}^{2}}\right)^{\frac{1}{2} \gamma\left(n, \alpha_{s}\right)}
$$

For the CF of "forward" operator (15) and two local operators we get

$$
\begin{aligned}
x_{23_{\perp}}^{2} & \left(\mu^{2} x_{23_{\perp}}^{2}\right)^{\gamma_{K}} \frac{1}{2 \pi i} \int d x_{1_{+}}\left\langle S_{1 n}\left(x_{1_{+}}, x_{1_{\perp}}\right) \mathcal{O}\left(x_{2_{-}}, x_{2_{\perp}}\right) \mathcal{O}\left(x_{3_{-}}, x_{3_{\perp}}\right)\right\rangle \\
= & -\frac{c_{n}\left[1+(-1)^{n}\right]}{x_{23_{\perp}}^{4}} \frac{\Gamma\left(1+2 n+\gamma_{n}\right)}{\Gamma^{2}\left(1+n+\frac{1}{2} \gamma_{n}\right)}\left(\frac{x_{23_{\perp}}^{2}}{x_{2-} x_{3-}}\right)^{1+\frac{\gamma_{n}}{2}}\left(\frac{x_{12_{\perp}}^{2}}{x_{2-}}+\frac{x_{13_{\perp}}^{2}}{x_{3-}}\right)^{-1-n-\gamma_{n}}
\end{aligned}
$$

Since a light-ray operator $S_{1 j}$ is an "analytical continuation" of a local operator $S_{1}^{n}$ to non-integer $n$ the $\mathrm{CF}$ of light-ray operator and two local operators has similar form

$$
\begin{aligned}
& x_{23_{\perp}}^{2}\left(\mu^{2} x_{23_{\perp}}^{2}\right)^{\gamma_{K}}\left\langle S_{1 j}\left(x_{1_{\perp}}\right) \mathcal{O}\left(x_{2_{-}}, x_{2_{\perp}}\right) \mathcal{O}\left(x_{3_{-}}, x_{3_{\perp}}\right)\right\rangle \\
& \quad=-2 \pi i \frac{c_{j}\left[1+e^{i \pi j}\right]}{x_{23_{\perp}}^{4}} \frac{\Gamma\left(1+2 j+\gamma_{j}\right)}{\Gamma^{2}\left(1+j+\frac{1}{2} \gamma_{j}\right)}\left(\frac{x_{23_{\perp}}^{2}}{x_{2-} x_{3-}}\right)^{1+\frac{\gamma_{j}}{2}}\left(\frac{x_{12_{\perp}}^{2}}{x_{2-}}+\frac{x_{13_{\perp}}^{2}}{x_{3-}}\right)^{-1-j-\gamma_{j}}
\end{aligned}
$$

Integrating this over the total translation in $x_{-}$direction we get

$$
\begin{aligned}
x_{23_{\perp}}^{2} & \left(\mu^{2} x_{23_{\perp}}^{2}\right)^{\gamma_{K}} \int d x_{3_{-}}\left\langle S_{1 j}\left(x_{1_{\perp}}\right) \mathcal{O}\left(L_{-}+x_{3_{-}}, x_{2_{\perp}}\right) \mathcal{O}\left(x_{3_{-}}, x_{3_{\perp}}\right)\right\rangle \\
= & \int_{0}^{1} d u \frac{c\left(j, \alpha_{s}\right)\left[1+e^{i \pi j}\right] L_{-}^{j}(\bar{u} u)^{j}}{\left[x_{12_{\perp}}^{2} u+x_{13_{\perp}}^{2} \bar{u}\right]^{1+j}}\left(\frac{\bar{u} u \mu^{-2} x_{23_{\perp}}^{2}}{\left[x_{12_{\perp}}^{2} u+x_{13_{\perp}}^{2} \bar{u}\right]^{2}}\right)^{\frac{1}{2} \gamma\left(j, \alpha_{s}\right)}
\end{aligned}
$$

To get the expansion of $\int d x_{3_{-}} \mathcal{O}\left(L_{-}+x_{3_{-}}, x_{2_{\perp}}\right) \mathcal{O}\left(x_{3_{-}}, x_{3_{\perp}}\right)$ as $x_{23_{\perp}} \rightarrow 0$ one compares Eq. (33) to the CF of two light-ray operators

$$
\left\langle S_{j=\frac{3}{2}+i \nu}\left(x_{1_{\perp}}\right) \tilde{S}_{j^{\prime}=\frac{3}{2}+i \nu^{\prime}}\left(x_{3_{\perp}}\right)\right\rangle=\frac{\delta\left(\nu-\nu^{\prime}\right) a\left(j, \alpha_{s}\right)}{\left(x_{13_{\perp}}^{2}\right)^{j+1}\left(x_{13_{\perp}}^{2} \mu^{2}\right)^{\gamma\left(j, \alpha_{s}\right)}}
$$

One obtains

$$
\begin{aligned}
x_{23_{\perp}}^{2}\left(\mu^{2} x_{23_{\perp}}^{2}\right)^{\gamma_{K}} \int d x_{3_{-}} \mathcal{O}\left(L_{-}+x_{2_{-}}, x_{2_{\perp}}\right) \mathcal{O}\left(x_{3_{-}}, x_{3_{\perp}}\right) \\
\quad=\int_{\frac{1}{2}-i \infty}^{\frac{1}{2}+i \infty} d j c\left(j, \alpha_{s}\right)\left[1+e^{i \pi j}\right] L_{-}^{j}\left(\mu^{2} x_{23_{\perp}}^{2}\right)^{\gamma\left(j, \alpha_{s}\right)} S_{1 j}\left(x_{3_{\perp}}\right)
\end{aligned}
$$

It is easy to see that the substitution of Eq. (34) to the r.h.s. of this equation reproduces the 3 -point CF (33). Similar result with exchange of $x_{-}$and $x_{+}$directions 


\section{Balitsky}

reads

$$
\begin{aligned}
x_{12_{\perp}}^{2} & \left(\mu^{2} x_{12_{\perp}}^{2}\right)^{\gamma_{K}} \int d x_{1_{+}} \mathcal{O}\left(L_{+}+x_{2_{+}}, x_{1_{\perp}}\right) \mathcal{O}\left(x_{2_{+}}, x_{2_{\perp}}\right) \\
\quad= & \int_{\frac{1}{2}-i \infty}^{\frac{1}{2}+i \infty} d j c\left(j, \alpha_{s}\right)\left[1+e^{i \pi j}\right] L_{+}^{j}\left(\mu^{2} x_{12_{\perp}}^{2}\right)^{\gamma\left(j, \alpha_{s}\right)} S_{j}^{+}\left(x_{1_{\perp}}\right)
\end{aligned}
$$

Finally, combining the light-cone expansion (36) with the three-point CF (33) we get the "DGLAP representation" of the 4-point CF (29)

$$
\begin{aligned}
& A\left(L_{+}, L_{-} ; x_{1_{\perp}}, x_{2_{\perp}}, x_{3_{\perp}}, x_{4_{\perp}}\right)=\int_{\frac{1}{2}-i \infty}^{\frac{1}{2}+i \infty} \frac{d j}{2 \pi i} c^{2}\left(j, \alpha_{s}\right) \\
& \quad \times \int_{0}^{1} d v \frac{\left[1+e^{i \pi j}\right]\left(L_{+} L_{-}\right)^{j}}{\left[x_{12_{\perp}}^{2} v+x_{13_{\perp}}^{2} \bar{v}\right]^{1+j}}\left(\frac{x_{12}^{2} x_{34}^{2}}{\left[x_{13_{\perp}}^{2} v+x_{14_{\perp}}^{2} \bar{v}\right]^{2}}\right)^{\frac{1}{2} \gamma\left(j, \alpha_{s}\right)}(\bar{v} v)^{j+\frac{1}{2} \gamma\left(j, \alpha_{s}\right)}
\end{aligned}
$$

In next Section we compare this formula with the "BFKL" representation of the same CF (29).

\section{BFKL Representation of 4-Point Correlation Function}

The 4-point CF (27) is a function of two conformal ratios. In the Regge limit (28) it is convenient to choose them as

$$
\begin{aligned}
R & =\frac{x_{13}^{2} x_{324}^{2}}{x_{12}^{2} x_{34}^{2}} \rightarrow \frac{\rho^{2} \rho^{\prime 2} x_{1+} x_{2+}+x_{3-} x_{4-}}{x_{12_{\perp}}^{2} x_{34 \perp}^{2}}, \quad r=R\left[1-\frac{x_{14}^{2} x_{23}^{2}}{x_{13}^{2} y_{24}^{2}}+\frac{1}{R}\right]^{2} \\
& \rightarrow \frac{\left[x_{24 \perp}^{2} x_{1+} x_{3-}+x_{2+} y_{4-} x_{12_{\perp}}^{2}+x_{1+} y_{4-}-x_{23_{\perp}}^{2}+x_{2+} x_{3-} x_{14 \perp}^{2}\right]^{2}}{x_{13_{\perp}}^{2} x_{24 \perp}^{2} x_{1+} x_{2+} x_{3-} x_{4-}}
\end{aligned}
$$

It is easy to see that $R$ increases with "energy" $\left(\sim \sqrt{\rho \rho^{\prime}}\right)$ while $r$ is energyindependent.

It was demonstrated that a 4 -point $\mathrm{CF}$ in $\mathcal{N}=4 \mathrm{SYM}$ in the Regge limit and at large $N_{c}$ can be parametrized as a contribution of a Regge pole with $J=\aleph(\nu)$

$$
A\left(x_{i}\right) \stackrel{s \sim \rho \rho^{\prime} \rightarrow \infty}{=} \frac{i}{2} \int d \nu f_{+}(\aleph(\lambda, \nu)) F(\lambda, \nu) \Omega(r, \nu) R^{\aleph(\lambda, \nu) / 2}
$$

where $f_{+}(\omega)=\frac{e^{i \pi \omega}-1}{\sin \pi \omega}$ is a signature factor and

$$
\Omega(r, \nu)=\frac{\nu}{2 \pi^{2}} \frac{\sin 2 \nu \rho}{\sinh \rho}, \quad \cosh \rho=\frac{\sqrt{r}}{2}
$$

is a solution of the Laplace equation in $H_{3}$ hyperboloid $\left(\partial_{H_{3}}^{2}+\nu^{2}+1\right) \Omega(r, \nu)=0$. The dynamics is described by the pomeron intercept $\aleph(\lambda, \nu)$ and the "pomeron residue" $F(\lambda, \nu)$. This formula was proved in Ref. [8] (see also [9]) by considering the leading Regge pole in a conformal theory. Also, it was demonstrated up to the NLO level that the structure (39) is reproduced by the high-energy OPE in Wilson lines. ${ }^{10-13}$ 
The pomeron intercept in $\mathcal{N}=4 \mathrm{SYM}$ is known in the leading order and in the NLO [14]

$$
\aleph(\nu)=\frac{\alpha_{s} N_{c}}{2 \pi}\left[\chi(\nu)+\frac{\alpha_{s} N_{c}}{4 \pi} \delta(\nu)\right]+O\left(\alpha_{s}^{3}\right)
$$

We will not need the explicit form of the "pomeron residue" $F(\lambda, \nu)$ but it can be easily restored from the NLO result for the CF of four protected operators $\operatorname{Tr} Z^{2}$ and $\operatorname{Tr} \bar{Z}^{2}$ calculated in Ref. [15].

Now let us take the DGLAP limit $x_{12 \perp}^{2} \rightarrow 0$ on the top of Regge limit (39). In this limit

$$
R=\frac{x_{13}^{2} x_{24}^{2}}{x_{12}^{2} x_{34}^{2}} \rightarrow \frac{x_{1_{+}} x_{2_{+}} x_{3_{-}} x_{4_{-}}}{x_{12_{\perp}}^{2} x_{34_{\perp}}^{2}}, \quad r \rightarrow \frac{x_{12_{+}}^{2}\left(x_{3_{-}} x_{14_{\perp}}^{2}-x_{4_{-}} x_{13_{\perp}}^{2}\right)^{2}}{x_{1_{+}} x_{2_{+}} x_{3_{-}} x_{4_{-}} x_{12_{\perp}}^{2} x_{34_{\perp}}^{2}}
$$

and

$$
\Omega(r, \nu) \rightarrow \frac{\nu}{2 \pi^{2} i}\left(r^{-\frac{1}{2}+i \nu}-r^{-\frac{1}{2}-i \nu}\right)
$$

so Eq. (39) reduces to

$$
\begin{aligned}
& A\left(L_{+}, L_{-} ; x_{1_{\perp}}, x_{2_{\perp}}, x_{3_{\perp}}, x_{4_{\perp}}\right)=\frac{i \alpha_{s}^{2}}{8} \pi^{2} L_{+} L_{-} \\
& \quad \times \int_{0}^{1} d u d v \int d \nu \frac{\tanh \pi \nu}{\nu \cosh ^{2} \pi \nu}\left(\frac{x_{12 \perp}^{2} x_{34 \perp}^{2} \bar{u} u \bar{v} v}{\left[x_{13 \perp}^{2} v+x_{14}^{2} \bar{v}\right]^{2}}\right)^{\frac{1}{2}+i \nu}\left(\frac{L_{+}^{2} L_{-}^{2} \bar{u} u \bar{v} v}{x_{12 \perp}^{2} x_{34 \perp}^{2}}\right)^{\aleph(\nu) / 2} f_{+}
\end{aligned}
$$

Performing integral over $u$ one obtaines the "BFKL" representation of the CF (29) in the double (Regge plus $x_{12 \perp}^{2} \rightarrow 0$ ) limit:

$$
\begin{aligned}
& A\left(L_{+}, L_{-} ; x_{1_{\perp}}, x_{2_{\perp}}, x_{3_{\perp}}, x_{4_{\perp}}\right)=\frac{\alpha_{s}^{2}}{8} \pi^{2} \int_{\frac{1}{2}-i \infty}^{\frac{1}{2}+i \infty} \frac{d \xi}{2 \pi i} f(\aleph(\xi))\left(L_{+} L_{-}\right)^{1+\aleph(\xi)} \\
& \times \int_{0}^{1} d v \frac{(\bar{v} v)^{1-\xi+\frac{\aleph(\xi)}{2}} \cos \pi \xi}{\left(\xi-\frac{1}{2}\right) \sin ^{3} \pi \xi} \frac{B\left(2-\xi+\frac{\aleph(\xi)}{2}\right)}{\left[x_{13 \perp}^{2} v+x_{14}^{2} \bar{v}\right]^{2+\aleph(\xi)}}\left(\frac{x_{12 \perp}^{2} x_{34 \perp}^{2}}{\left[x_{13 \perp}^{2} v+x_{14}^{2} \bar{v}\right]^{2}}\right)^{-\xi-\frac{\aleph(\xi)}{2}}
\end{aligned}
$$

where $\xi \equiv \frac{1}{2}+i \nu$.

\section{Conclusion: Anomalous Dimensions of Light-Ray Operators}

Let us compare integrals (37) and(43) for the correlation function (27). In both cases, the integrals run parallel to the imaginary axis but the singularities of the integrands lie on the negative real axis. We can close the integrals around negative real axis and compare the integrands near $j=0$ and $\xi=0$. We see that the 


\section{Balitsky}

integrands coincide if one makes an identification

$$
1+\aleph\left(\xi, \alpha_{s}\right)=j, \quad \gamma\left(j, \alpha_{s}\right)=-2 \xi-\aleph(\xi)
$$

and

$$
c^{2}\left(j, \alpha_{s}\right)=\frac{\alpha_{s}^{2}}{8} \pi^{2} \frac{\cos \pi \xi}{\left(\xi-\frac{1}{2}\right) \sin ^{3} \pi \xi} \frac{\Gamma^{2}\left(2-\xi+\frac{\aleph(\xi)}{2}\right)}{\Gamma(4-2 \xi+\aleph(\xi))} \frac{\partial j}{\partial \xi}
$$

If we write down the NLO pomeron intercept (41) as a Laurent series near $\xi=0$

$$
\aleph\left(\xi, \alpha_{s}\right)=\frac{\alpha_{s} N_{c}}{\pi \xi}+\ldots
$$

we can invert equations (44) and get anomalous dimension near $\omega=j-1 \rightarrow 0$ as a series in $\frac{\alpha_{s}}{\omega}$

$$
\begin{aligned}
& \aleph(\xi)-2 \aleph(\xi) \aleph^{\prime}(\xi) \simeq \frac{\alpha_{s} N_{c}}{\pi \xi}+\frac{\zeta(3) \alpha_{s}^{2}}{\xi}+\ldots \\
& \left.\Rightarrow \gamma_{j}=-2 \frac{\alpha_{s} N_{c}}{\pi \omega}+[0+\zeta(3) \omega]\left(\frac{\alpha_{s} N_{c}}{\pi \omega}\right)\right)^{3}+\ldots
\end{aligned}
$$

In principle, the inversion of Eqs. (44) gives all orders in $\left(\frac{\alpha_{s} N_{c}}{\pi \omega}\right)^{n}$ but in practice one gets the first few terms since the analytical form of the inversion is not known.

It is worth noting that the second of Eqs. (44) corresponds to the shift (5)

$$
\xi \rightarrow \xi+\frac{1}{2} \aleph(\xi)
$$

in the integral (43). In the momentum space this shift comes from the change of the energy scale from the symmmetric $Q P$ to non-symmetric $Q^{2}$ while in the coordinate space it comes directly from the symmetric "energy scale" $R$, see Eq. (38).

This work was supported by contract DE-AC05-06OR23177 under which the Jefferson Science Associates, LLC operate the Thomas Jefferson National Accelerator Facility.

\section{References}

1. V.S. Fadin, E.A. Kuraev, and L.N. Lipatov, Sov.Phys.JETP 44, 443-450 (1976), Sov.Phys.JETP 45, 199 (1977);

I. Balitsky and L.N. Lipatov, Sov. Journ. Nucl. Phys. 28, 822 (1978).

2. T. Jaroszewicz, Phys.Lett.B116, 291(1982).

3. V.S. Fadin and L.N. Lipatov, Phys. Lett.B429, 127 (1998);

G. Camici and M. Ciafaloni, Phys. Lett.B430, 349 (1998).

4. A. Vogt, S. Moch, and J.A.M. Vermaseren, Nucl.Phys. B691, 129 (2004).

5. T. Lukowski, A. Rej, and V.N. Velizhanin, Nucl.Phys. B831, 105 (2010).

6. I. Balitsky and V. M. Braun, Nucl.Phys. B311, 541 (1989).

7. A.V. Belitsky, S.E. Derkachov, G.P. Korchemsky, and A.N. Manashov, Phys.Rev. D70, 045021(2004).

8. L. Cornalba, "Eikonal methods in AdS/CFT: Regge theory and multi-reggeon exchange", arXiv:0710.5480 [hep-th] (2007).

9. M.S. Costa, V. Goncalves, and J. Penedones, JHEP 1212, 091 (2012). 
10. I. Balitsky, Nucl. Phys. B463, 99 (1996);

"Operator expansion for diffractive high-energy scattering", [hep-ph/9706411].

11. I. Balitsky, Phys. Rev. D60, 014020 (1999).

12. I. Balitsky, "High-Energy QCD and Wilson Lines", In *Shifman, M. (ed.): At the frontier of particle physics, vol. 2*, p. 1237-1342 (World Scientific, Singapore, 2001) [hep-ph/0101042].

13. I. Balitsky, "High-Energy Ampltudes in the Next-to-Leading Order", In "Subleties in Quantum Field Theory", ed D. Diakonov, (PNPI Publishing Dept., 2010); arXiv:1004.0057 [hep-ph]

14. A.V. Kotikov, L.N. Lipatov, Nucl. Phys.B 58219 (2000); Nucl. Phys.B 661, 19 (2003); Nucl. Phys.B 685, 405 (2004) (Erratum).

15. I. Balitsky and G.A. Chirilli, Phys.Lett. B687, 204 (2010). 